\title{
Effect of infusion tests on the dynamical properties of intracranial pressure in hydrocephalus
}

María García ${ }^{a}$, Jesús Poza ${ }^{\text {a,b,c }}$, Alejandro Bachiller ${ }^{\text {a }}$, David Santamarta ${ }^{\text {d, and Roberto }}$ Hornero $^{\text {a,b }}$

${ }^{a}$ Biomedical Engineering Group, Department T.S.C.I.T., E.T.S. Ingenieros de Telecomunicación, University of Valladolid, Valladolid, Spain

${ }^{\mathrm{b}}$ IMUVA, Instituto de Investigación en Matemáticas, University of Valladolid, Valladolid, Spain ${ }^{\mathrm{c}}$ INCYL, Instituto de Neurociencias de Castilla y León, University of Salamanca, Salamanca, Spain ${ }^{\text {d }}$ Servicio de Neurocirugía, Hospital Universitario de León, León, Spain

Address correspondence to:

\section{María García Gadañón}

Biomedical Engineering Group (GIB), Department T.S.C.I.T., E.T.S. Ingenieros de Telecomunicación, University of Valladolid, Paseo de Belén 15, 47011, Valladolid, Spain Phone: +34983423983

Fax: +34 983423667

Electronic mail: maria.garcia@tel.uva.es

URL: www.gib.tel.uva.es 


\section{ABSTRACT}

Background and Objective: Hydrocephalus comprises a number of conditions characterised by clinical symptoms, dilated ventricles and anomalous cerebrospinal fluid (CSF) dynamics. Infusion tests (ITs) are usually performed to study CSF circulation and in the preoperatory evaluation of patients with hydrocephalus. The study of intracranial pressure (ICP) signals recorded during ITs could be useful to gain insight into the underlying pathophysiology of this condition and to further support treatment decisions. In this study, two wavelet parameters, wavelet turbulence $(W T)$ and wavelet entropy $(W E)$, were analysed in order to characterise the variability, irregularity and similarity in spectral content of ICP signals in hydrocephalus.

Methods: One hundred and twelve ICP signals were analysed using WT and WE. These parameters were calculated in two frequency bands: $B_{1}(0.15-0.3 \mathrm{~Hz})$ and $B_{2}(0.67-2.5$ $\mathrm{Hz}$ ). Each signal was divided into four artifact-free epochs corresponding to the basal, early infusion, plateau and recovery phases of the IT. We calculated the mean and standard deviation of $W T$ and $W E$ and analysed whether these parameters revealed differences between epochs of the IT.

Results: Statistically significant differences $\left(p<1.7 \cdot 10^{-3}\right.$, Bonferroni-corrected Wilcoxon signed-rank tests) in pairwise comparisons between phases of ITs were found using the mean and standard deviation of $W T$ and $W E$. These differences were mainly found in $B_{2}$.

Conclusions: Wavelet parameters like $W T$ and $W E$ revealed changes in the signal time-scale representation during ITs. Statistically significant differences were mainly found in $B_{2}$, associated with ICP pulse waves, and included a higher degree of similarity in the spectral content, together with a lower irregularity and variability in the plateau phase with respect to the basal phase. 
Keywords - Hydrocephalus, infusion test, intracranial pressure, wavelet entropy, wavelet turbulence. 


\section{INTRODUCTION}

Adult hydrocephalus encompasses a heterogeneous group of disorders occurring in a wide range of ages, severity of symptoms and physiological states [1]. Patients with hydrocephalus generally show clinical symptoms, ventriculomegaly and anomalous cerebrospinal fluid (CSF) dynamics [1,2]. Normal pressure hydrocephalus (NPH) can appear as a primary condition [3] or as a consequence of subarachnoid haemorrhage, traumatic brain injury (TBI) or meningitis [1,3]. Implantation of a CSF shunt is the main treatment option [4]. However, not all patients improve after surgery and their condition becomes challenging for neurosurgeons [1,5]. Despite the recent advances, treatment is sometimes based on a limited knowledge of the underlying pathophysiology [6]. Therefore, the study of intracranial pressure (ICP) and CSF dynamics can provide valuable information on the management of patients with hydrocephalus [7].

Lumbar infusion tests (ITs) are frequently performed in the preoperatory evaluation of subjects who show features of NPH [8]. In ITs, ICP is artificially raised by the injection of fluid in the lumbar CSF space. Then, pressure is recorded and the resistance to CSF outflow is calculated [8]. Additionally, the applications of ITs include the assessment of shunt function [9], the analysis of metabolic changes in periventricular white matter [10] and the study of the haemodynamic response associated with ICP [11].

Traditionally, therapies derived from ICP monitoring relied on the time-averaged mean, as this parameter has been related to pathological patterns [12]. However, it does not account for all the information contained in the ICP waveform and does not clarify the pathophysiology underlying NPH $[8,12]$. For this reason, prior research has been devoted to the development of alternatives to study ICP. Some of them were based on non-linear methods, including approximate entropy [13], multiscale entropy [14] and Lempel-Ziv complexity [8,15]. Results revealed that intracranial hypertension was associated with a lower ICP signal complexity in 
children with TBI [13,15] and in adults with hydrocephalus [8]. Moreover, reduced complexity seems to be linked to poor outcome after TBI [14]. On the other hand, previous studies addressed the spectral analysis of ICP signals [11,16-18]. Thereby, the reconstruction of an ICP signal from its first harmonic was accomplished [17]. The relationship between resistance to CSF outflow and three spectral components of the ICP waveform was also analysed [11]. Finally, the study of very low frequency components of the ICP signal (slow waves) has also received interest [16]. In our previous research, we addressed the spectral analysis of ICP recordings from ITs using median frequency and relative power [18]. The morphology of ICP pulse waveforms has also been analysed to characterise ICP dynamics [19-21]. The Morphological Clustering and Analysis of ICP Pulse (MOCAIP) algorithm has been proposed to detect ICP pulse waveform peaks based on ICP and electrocardiographic (ECG) signals [20]. This approach has also been used in a later study to determine whether the morphology of ICP pulse waves could be helpful to detect slow waves in overnight recordings [19]. Besides, the relationship between the shape of intracranial pulse waves and brain compliance has been analysed in the control of an hydrocephalus shunt [21].

Recent studies proposed an alternative spectral representation of ICP signals using the wavelet transform. This is a suitable methodology due to the non-linear, non-stationary and multiscale aspects of cerebral haemodynamics [22]. In this sense, some authors used the wavelet transform to analyse the instantaneous phase difference between arterial blood pressure (ABP) and ICP [22]. The wavelet spectrograms have been also analysed in long-term ICP recordings and ITs [23]. Parameters like windowed wavelet entropy and relative wavelet entropy, have been also used to study ICP signal irregularity in hypertension patients [24].

The present study represents a novel approach to analyse the effectiveness of several wavelet-based measures in characterising the variability, irregularity and similarity in spectral content of ICP signals in hydrocephalus. Initially, a time-scale representation of ICP signals 
was obtained by means of the continuous wavelet transform (CWT). Then, two parameters were calculated: wavelet turbulence $(W T)$ and wavelet entropy $(W E)$. They were analysed in two frequency bands: $B_{1}(0.15-0.3 \mathrm{~Hz})$, related to respiratory blood pressure oscillations [25]; and $B_{2}(0.67-2.5 \mathrm{~Hz})$, related to ICP pulse waves [25]. Thus, we tried to address the following research questions: (i) Are the proposed parameters useful to analyse the dynamical properties of ICP signals recorded during ITs?; and (ii) Can the proposed parameters be useful to evaluate the influence of respiratory and pulse waves of the ICP waveform in NPH?

\section{MATERIALS AND METHODS}

\subsection{Patients}

A database of 112 ICP signals recorded during ITs at the Department of Neurosurgery of the University Hospital of León (Spain) was analysed. The recordings belonged to patients suffering from hydrocephalus (65 male and 47 female, age $74 \pm 14$ years, mean \pm standard deviation, SD). Ventriculomegaly was observed in all patients (Evans index $\geq 0.30$ ). Participants presented different combinations of Hakim's triad: gait disturbances, cognitive

deterioration and urinary incontinence [9]. Lumbar ITs were performed as a supplementary hydrodynamic study to help in the decision on the surgical management of patients [8]. Table 1 summarises the data of the population under study.

All patients or a close relative gave their informed consent to be included in the study. The study was approved by the Ethics Committee at the University Hospital of León (Spain).

\section{INSERT TABLE 1 AROUND HERE}

\subsection{Data acquisition protocol}

ITs were performed using a variant of the Katzman and Hussey method [8]. Patients laid in the lateral recumbent position and, under local anaesthesia, two needles were inserted in their 
lower lumbar region. Infusion was performed through a caudal needle with an infusion pump (Lifecare ${ }^{\circledR} 5000$, Abbott Laboratories) connected to the needle through a three-way stopcock. Pressure measurement was performed through a rostral needle connected to a pressure microtransducer $\left(\right.$ Codman $^{\circledR}$ MicroSensor $^{\mathrm{TM}}$ ICP transducer, Codman \& Shurtleff). The analogue output of the microtransducer was connected to an amplifier (ML110 Bridge amplifier), an analogue to digital converter (PowerLab 2/25 Data recording system ML825, ADI Instruments) and a computer, where signals were displayed and recorded $[8,18]$.

Data were recorded continuously and four phases could be identified in ITs. Firstly, the baseline pressure $\left(P_{0}\right)$ was registered for approximately 5 minutes. Then, a Ringer solution was infused at a constant rate of $1.5 \mathrm{ml} / \mathrm{min}$. The infusion ceased when a plateau was reached in the pressure levels. At this point, the plateau pressure $\left(P_{p}\right)$ was measured. CSF pressure was still recorded after infusion stopped, until it decreased towards baseline levels $[8,18]$. A qualified neurosurgeon visually selected four artefact-free epochs in each recording $[8,18]$ :

- Epoch $0\left(E_{0}\right)$ was representative of the basal phase of the IT, for which $P_{0}$ was measured.

- Epoch $1\left(E_{l}\right)$ corresponded to the early infusion phase, where ICP recordings usually described an ascending slope.

- Epoch $2\left(E_{2}\right)$ represented the plateau phase. $P_{p}$ was obtained in this stage.

- Epoch $3\left(E_{3}\right)$ was connected to the recovery phase, where the pressure signal slowly decreased.

Fig. 1 depicts one ICP recording in our database, where the four artefact-free epochs have been indicated. 
The sampling frequency $\left(f_{s}\right)$ for data acquisition was $100 \mathrm{~Hz}$. Furthermore, ICP signals were filtered using a finite impulse response (FIR) band-pass filter with cutoff frequencies 0.02 and $5 \mathrm{~Hz}$. These frequency limits ensured that the meaningful spectral content of the recordings was preserved, while the DC component was minimised [18].

\subsection{Continuous wavelet transform $(C W T)$}

CWT can be used to analyse time series with a variable resolution in the time-frequency plane [26]. It is an adequate tool in the context of ICP signals due to the nonlinear, nonstationary and multiscale features of cerebral haemodynamics [22]. CWT is based on decomposing the signal to be analysed, $x(t)$, using translated and dilated versions of a function called mother wavelet, $\psi(x)$ [26]. The wavelet coefficients, $W(\tau, s)$, represent the similitude between $x(t)$ and the scaled and shifted versions of $\psi(x)$ [26].

A wavelet is a zero-mean function that is localized in both time and frequency $[27,28]$. Many different waveforms could be used as mother wavelet [26]. In this study, we chose the complex Morlet wavelet. It is a Gaussian-windowed sinusoidal function with several cycles [29], which has been previously used in the analysis of several types of biological signals that show a non-stationary behaviour [22,30-32], including ICP signal analysis [22,24]. It is defined as [22]:

$$
\psi(t)=\frac{1}{\sqrt{\pi \cdot \Omega_{b}}} \cdot \exp \left(j 2 \pi \Omega_{c} t\right) \cdot \exp \left(\frac{-t^{2}}{\Omega_{b}}\right),
$$

where $\Omega_{b}$ is the bandwidth parameter and $\Omega_{c}$ is the wavelet centre frequency. In this study, both parameters were set to 1 , in order to obtain a good trade-off between time resolution $(\Delta t)$ and frequency resolution $(\Delta f)$ at low frequencies [22].

The relationship between $\Delta t$ and $\Delta f$ is important in wavelet analysis. According to the Heisenberg uncertainty principle, it is not possible to achieve arbitrarily good resolution in 
time and frequency simultaneously [27]. Thus, CWT provides good $\Delta t$ at high frequencies and good $\Delta f$ at low frequencies [26]. This issue was considered in the calculation of the CWT, by defining a Heisenberg box. It is a rectangle centred at each point in the timefrequency plane, whose width depends on the time and frequency resolution [28]. In addition, it should be noticed that the four artefact-free epochs analysed for each ICP recording are finite short-time signals. This means that CWT calculation would be affected by edge effects at the beginning and end of each epoch [27]. To take this problem into account, a cone of influence (COI) can be defined based on the Heisenberg box approach. The COI delimitates the region in the time-frequency plane in which edge effects can be ignored [27]. In this study, a COI was established for each of the four artefact-free epochs. The width of the Heisenberg box was chosen to be $2 \cdot \Delta t$ by $2 \cdot \Delta f$ [30]. Fig. 2 shows the scalogram and COIs corresponding to the artefact-free epochs of the ICP recording in Fig. 1.

The ICP recordings in our database were analysed in two frequency bands. $B_{1}$ corresponded to a frequency range between 0.15 and $0.3 \mathrm{~Hz}$ (9-18 cycles per minute) and is related to respiratory blood pressure oscillations [25]. $B_{2}$ corresponded to a frequency range between 0.67 and $2.5 \mathrm{~Hz}$ (40-150 cycles per minute) and is related to ICP pulse waves [25]. Only those CWT coefficients whose associated Heisenberg boxes were completely included in the COI were taken into account [30].

\section{DISPLAY FIGURE 2 AROUND HERE}

\subsection{Wavelet turbulence (WT)}

$W T$ has been previously used in the analysis of biomedical recordings to quantify the spectral changes of a signal over time [33]. It is based on calculating the correlation coefficient between adjacent time segments of the CWT, as a measure of the similitude between these segments [31]. It can be defined as: 


$$
W T(\tau)=\rho[W(\tau, s), W(\tau+1, s)], \quad \tau=1, \ldots, N_{T}-1,
$$

where $\rho[\cdot]$ denotes the Pearson correlation coefficient between $W(\tau, s)$ and $W(\tau+1, s), s$ represents the scale and $N_{T}$ is the signal length.

The mean $(\langle W T\rangle)$ and the standard deviation $(\mathrm{SD}[W T])$ were subsequently calculated from the time series formed by the temporal evolution of $W T$ in the scales corresponding to frequency bands $B_{1}$ and $B_{2}$. $\langle W T\rangle$ summarizes the average degree of similarity between the spectral content of adjacent time slices, while $\mathrm{SD}[W T]$ describes the lack of homogeneity in correlation around the mean value [31]. An average value of $\langle W T\rangle$ and $\mathrm{SD}[W T]$ was obtained for each artefact-free epoch and frequency band. We will denote by $\left\langle W T_{B_{i}}^{E_{j}}\right\rangle$ the average value of $W T$ in epoch $E_{j}(j=0,1,2,3)$ and band $B_{i}(i=1,2)$. Similarly, $\operatorname{SD}\left[W T_{B_{i}}^{E_{j}}\right]$ denotes de value of $\operatorname{SD}[W T]$ in epoch $E_{j}(j=0,1,2,3)$ and band $B_{i}(i=1,2)$.

\subsection{Wavelet entropy (WE)}

Shannon's entropy $(S E)$ represents a disorder measure, which can be used to study the irregularity of signals in terms of the distribution of the spectral power along the different frequencies [34]. In the case that $S E$ is calculated from a wavelet decomposition of the signal, it is also known as wavelet entropy $(W E)$. The calculation of WE is based on the normalized wavelet scalogram $\left(W S_{n}\right)$, which is computed from the coefficients of the CWT as [30]:

$$
W S_{n}(\tau, s)=\frac{|W(\tau, s)|^{2}}{\sum_{s}|W(\tau, s)|^{2}}, \quad \tau=1, \ldots, N_{T}-1, \quad s \in S_{B_{i}}(i=1,2),
$$

where $S_{B_{i}}$ is the set of scales corresponding to the frequencies in band $B_{i}(i=1,2)$.

The calculation of WE can be carried out using the definition of Shannon's entropy [24]:

$$
W E(\tau)=-\frac{1}{\left|S_{B_{i}}\right|} \sum_{s \in S_{B_{i}}} W S_{n}(\tau, s) \cdot \ln \left[W S_{n}(\tau, s)\right], \quad \tau=1, \ldots, N_{T}-1,
$$


where $\left|S_{B_{i}}\right|$ denotes the cardinality of the set of scales corresponding to band $B_{i}(i=1,2)$.

Then, the mean $(\langle W E\rangle)$ and the standard deviation $(\mathrm{SD}[W E])$ were calculated from the time series formed by the temporal evolution of $W E$ in the scales corresponding to bands $B_{1}$ and $B_{2} .\langle W E\rangle$ summarises the average degree of temporal irregularity, while $\mathrm{SD}[W E]$ describes the variability in $W E$ around the mean value [35]. An average value of $\langle W E\rangle$ and $\mathrm{SD}[W E]$ was obtained for each artefact-free epoch, frequency band and ICP recording. We will denote by $<W E_{B_{i}}^{E_{j}}>$ to the average value of $W E$ in epoch $E_{j}(j=0,1,2,3)$ and band $B_{i}$ $(i=1,2)$. Similarly, $\operatorname{SD}\left[W E_{B_{i}}^{E_{j}}\right]$ denotes the average value of $\operatorname{SD}[W E]$ in epoch $E_{j}(j=0,1,2$, 3) and band $B_{i}(i=1,2)$.

\subsection{Statistical analysis}

An exploratory analysis was initially performed to analyse the data distribution. The Kolmogorov-Smirnov with Lilliefors significance correction and the Shapiro-Wilk tests were used to assess the normality of the wavelet parameters in the 4 artefact-free epochs. Our data did not meet parametric test assumptions. Therefore, the existence of statistically significant interactions $(\alpha=0.01)$ among epochs of the IT was assessed using the non-parametric Friedman test [36]. When statistically significant interactions were found, post hoc analyses were performed by means of the Wilcoxon signed-rank test with Bonferroni correction to account for multiple comparisons $\left(\alpha=0.01 / 6=1.7 \cdot 10^{-3}\right)[36]$.

Finally, the Spearman's correlation of wavelet parameters with patient data was analysed. The significance level considered was $\alpha=0.01$.

\section{RESULTS}

Table 2 summarises the median and interquartile range (IQR) of CSF pressure and epoch duration. These values were averaged over the 112 subjects in our database. 


\subsection{WT results}

The results of the Friedman test revealed significant interactions among phases of the IT

$$
\begin{aligned}
& \text { using }<W T_{B_{1}}>\left(\chi^{2}(3)=25.15, p=1.44 \cdot 10^{-5}\right), \operatorname{SD}\left[W T_{B_{1}}\right]\left(\chi^{2}(3)=27.29, p=5.12 \cdot 10^{-6}\right), \\
& <W T_{B_{2}}>\left(\chi^{2}(3)=106.75, p=5.50 \cdot 10^{-23}\right) \text { and } \operatorname{SD}\left[W T_{B_{2}}\right]\left(\chi^{2}(3)=86.92, p=1.00 \cdot 10^{-18}\right) .
\end{aligned}
$$

The Wilcoxon signed-rank test with Bonferroni correction was used to perform post hoc analyses of these interactions. Statistically significant differences were found between several pairs, as summarised in Table 3.

\section{INSERT TABLE 3 AROUND HERE}

Additionally, the evolution of $\left\langle W T_{B_{i}}^{E_{j}}>\right.$ and $\operatorname{SD}\left[W T_{B_{i}}^{E_{j}}\right](j=0,1,2,3$ and $i=1,2)$ along the IT was analysed (see Fig. 3). $<W T_{B_{1}}>$ values were very similar in all epochs for band $B_{l}$, while $\mathrm{SD}\left[W T_{B_{1}}\right]$ values were lower in $E_{0}$, increased during infusion reaching the highest values in $E_{2}$ and then decreased again in $E_{3}$. The results in $B_{2}$ followed a different trend. The minimum $<W T_{B_{2}}>$ values were found in the basal phase, these values then increased during infusion until the highest levels corresponding to the plateau phase. $\left\langle W T_{B_{2}}\right\rangle$ slightly decreased again in the recovery phase. Regarding $\operatorname{SD}\left[W T_{B_{2}}\right]$, the highest values corresponded to the basal phase. Then $\operatorname{SD}\left[W T_{B_{2}}\right]$ decreased during infusion, reaching the lowest levels in the plateau phase. The values slightly increased again in $E_{3}$. 


\subsection{WE results}

The non-parametric Friedman test showed significant interactions among phases of the IT using $<W E_{B_{1}}>\left(\chi^{2}(3)=15.91, p=1.18 \cdot 10^{-3}\right), \quad<W E_{B_{2}}>\left(\chi^{2}(3)=55.70, p=4.86 \cdot 10^{-12}\right)$ and $\operatorname{SD}\left[W E_{B_{2}}\right]\left(\chi^{2}(3)=108.03, p=2.91 \cdot 10^{-23}\right)$. Post hoc analyses were subsequently performed to analyse these interactions. Statistically significant differences were only detected in frequency band $B_{2}$, as shown in Table 3 .

Besides, the temporal evolution of $\left\langle W E_{B_{i}}^{E_{j}}>\right.$ and $\operatorname{SD}\left[W E_{B_{i}}^{E_{j}}\right](j=0,1,2,3$ and $i=1,2)$ is depicted in Fig. 4. The lowest $\left\langle W E_{B_{1}}>\right.$ was found in the basal phase, then increased during infusion and reached the highest levels in the plateau phase. Finally, $\left\langle W E_{B_{1}}\right\rangle$ slightly decreased in the recovery phase. The values in $\operatorname{SD}\left[W E_{B_{1}}\right]$ were very similar in the four phases of the IT. The tendency in frequency band $B_{2}$ was similar using $<W E_{B_{2}}>$ and $\operatorname{SD}\left[W E_{B_{2}}\right]$. The values were higher in $E_{0}$, decreased during infusion to reach the lowest values in $E_{2}$ and then slightly increased again in $E_{3}$.

\section{DISPLAY FIGURE 4 AROUND HERE}

\subsection{Correlation with patient data}

We also wanted to know whether the spectral parameters could reveal any relationship between phases of the infusion tests and some demographic, radiological and ICP-based variables recorded for the 112 patients in our database. They included: age, Evans index $(E)$, basal pressure of the CSF pressure signal $\left(P_{0}\right)$, basal amplitude of the CSF pressure signal $\left(A_{0}\right)$, plateau pressure of the CSF pressure signal $\left(P_{p}\right)$, plateau amplitude of the CSF pressure signal $\left(A_{p}\right)$, CSF outflow resistance $\left(R_{O U T}\right)$ and an estimated pressure-volume compensatory reserve index (RAP index). 
In the case of the RAP index, it was calculated as the correlation coefficient between mean ICP and the estimated amplitude of the first harmonic of the pulse waveform (AMP) during the first 4 minutes of the recording. For this task, the signal was divided into 6-s length nonoverlapping windows $[2,12,37]$. In each 6-s length window, mean ICP was calculated and AMP was estimated as the squared root of the power of the first harmonic component [38]. Therefore, 40 consecutive data points were considered in the calculation of the correlation coefficient $[2,12,37]$.

The correlation results can be seen in Table 4. In order to simplify this table, only the correlation results for $A_{0}, A_{p}, R_{O U T}$ and the RAP index were included. The reason is that, for $A_{0}$ and $A_{p}$, the most significant correlations where found, while $R_{O U T}$ and the RAP index are the parameters most linked to compliance. In this table, some statistically significant correlations between wavelet parameters and $A_{0}, A_{p}$ and $R_{O U T}$ can be found. The most significant correlations appeared for the correlations between $\left\langle W T_{B_{2}}\right\rangle$ and $A_{p}$ and for the correlations between $\mathrm{SD}\left[W T_{B_{2}}\right]$ and $A_{p}$.

\section{INSERT TABLE 4 AROUND HERE}

\section{DISCUSSION}

In this study, the changes produced in $W T$ and $W E$ during the phases of ITs in patients with hydrocephalus were analysed. Specifically, we have explored the changes in $W T$ and $W E$ during ITs in frequency bands $B_{1}(0.15-0.3 \mathrm{~Hz})$ and $B_{2}(0.67-2.5 \mathrm{~Hz})$, associated with the respiratory and the pulse components of the ICP waveform, respectively [25].

\subsection{Dynamical properties of ICP recordings}

Regarding the first research question, results for $W T$ in Table 3 revealed that nonsignificant differences between phases of the IT were detected using $<W T_{B_{1}}>$. However, we 
found a significant increase in $\mathrm{SD}\left[W T_{B_{1}}^{E_{2}}\right]$ with respect to $\mathrm{SD}\left[W T_{B_{1}}^{E_{0}}\right]$ and in $\operatorname{SD}\left[W T_{B_{1}}^{E_{2}}\right]$ with respect to $\mathrm{SD}\left[W T_{B_{1}}^{E_{3}}\right]$. These findings suggest that there is a significantly higher variability in the spectral content of band $B_{1}$ when CSF pressure reaches the range of intracranial hypertension. In the case of band $B_{2}$, we found significant differences between the basal phase and the remaining phases of the ITs using $\left\langle W T_{B_{2}}>\right.$ and $\operatorname{SD}\left[W T_{B_{2}}\right]$. It should be noted that our results showed a significant increase in $\left\langle W T_{B_{2}}^{E_{2}}\right\rangle$ with respect to $\left\langle W T_{B_{2}}^{E_{0}}\right\rangle$. This result suggests an increase in the average degree of similarity in the spectral content during the state of intracranial hypertension when compared with the resting state. However, these spectral changes appear only in $B_{2}$, indicating that this similarity increase is mainly associated to the pulse waves [11]. Furthermore, $\langle W T\rangle$ can be considered as an indirect measure of signal irregularity [35]. In this sense, the aforementioned results suggest an irregularity loss in $E_{2}$ with respect to $E_{0}$. In previous studies, a decrease in Lempel-Ziv (LZ) complexity in the plateau phase of ITs with respect to the basal phase was reported [8]. Reduced complexity was also found in paediatric patients suffering from traumatic brain injury and intracranial hypertension [15]. Certainly, complexity and irregularity are complementary measures that quantify the degree of disorder in the different phases of ITs. It should also be stressed that a significant decrease in $\operatorname{SD}\left[W T_{B_{2}}^{E_{2}}\right]$ with respect to $\operatorname{SD}\left[W T_{B_{2}}^{E_{0}}\right]$ was found. This result can be associated with a loss of variability in the plateau phase when compared with the basal phase.

Regarding $W E$, the differences between phases of the IT were not statistically significant for frequency band $B_{1}$. However, results in Table 3 indicate that significant differences between the basal phase and the remaining phases of the IT could be detected in $B_{2}$, using both $<W E_{B_{2}}>$ and $\mathrm{SD}\left[W E_{B_{2}}\right]$. Besides, we also found statistically significant differences between $E_{1}$ and $E_{3}$ using $\operatorname{SD}\left[W E_{B_{2}}\right]$. Remarkably, a statistically significant decrease in 
$<W E_{B_{2}}^{E_{2}}>$ with respect to $\left\langle W E_{B_{2}}^{E_{0}}>\right.$ was detected. This result suggests that the spectral distribution in the hypertension state is less irregular than in the resting state [39]. These findings for band $B_{2}$ are concordant with the results obtained with $W T$. As formerly stated, similar results were found using complexity measures in previous studies [8,15]. It should also be stressed that a significant decrease in $\mathrm{SD}\left[W E_{B_{2}}^{E_{2}}\right]$ with respect to $\mathrm{SD}\left[W E_{B_{2}}^{E_{0}}\right]$ was found. These results suggest that intracranial hypertension due to volume loading produces a significantly lower variability in the spectral content of band $B_{2}$. This variability decrease could be linked to the decrease in data dispersion found in previous studies on ITs, measured in terms of the standard deviation of LZ values [8]. In this study, the SD in LZ complexity values was lower in the plateau phase than in the basal phase. Oppositely, other studies reported an increased variability in the ICP signal during the plateau phase of ITs [40]. However, variability was measured in terms of data dispersion using central tendency measure (CTM). $\mathrm{SD}[W E]$ differs from CTM, since it quantifies temporal variability as the homogeneity in $W E$ values along phases of the IT.

\subsection{Respiratory and pulse-driven abnormalities in $N P H$}

The second research question addresses the influence of the components of the ICP waveform. In this sense, the differences in the average degree of similarity, irregularity and variability found in $E_{2}$ with respect to $E_{0}$ could be related to different effects. In the first place, NPH has been associated with low intracranial compliance [41]. Vascular compliance is defined as the rate of change in the vascular volume with respect to pressure changes [41]. It is important because the brain is surrounded by an incompressible fluid (CSF) and enclosed in a rigid container (the skull) [41]. ICP oscillates during the cardiac cycle as a result of cardiac-driven variations in $\mathrm{ABP}$ [42]. The blood volume entering the brain changes within the cardiac cycle, resulting in a net intracranial inflow of blood during systole and net outflow 
during diastole [41]. These blood volume changes during the cardiac cycle must be compensated by the CSF volume changes in order to maintain a stable ICP, leading to ABPdriven pulsations in CSF pressure [41]. However, vascular compliance seems to be reduced in disorders such as NPH [42]. Besides, it has been suggested that volume load during ITs may also have a relevant impact in the compliance of the brain and cerebral blood vessels. This issue leads to an exhausted compensatory reserve in the plateau phase of ITs, independently of the pathogenesis of hydrocephalus [7,43]. In this study, we found a decrease in irregularity and variability, together with a higher degree of similarity in the spectral content of the ICP waveform in the plateau phase with respect to the basal phase. These changes were mainly observed in band $B_{2}$, related to pulse waves [25]. Therefore, they might be related to modifications in the ICP waveform associated with reduced brain compliance in NPH or with hypertension induced by ITs. Additional measures would be needed to confirm this relationship.

In this sense, it has been suggested that a reduced brain compliance may be associated with distinctive morphological patterns of the ICP pulse waves [21,44]. Therefore, the shape of ICP pulse waves may change during ITs, from patterns representing undistorted compliance to patterns representing reduced brain compliance. This shape change of pulse waves may be connected with the differences found in $<W T_{B_{2}}>, \operatorname{SD}\left[W T_{B_{2}}\right],<W E_{B_{2}}>$ and $\operatorname{SD}\left[W E_{B_{2}}\right]$, especially when comparing $E_{2}$ and $E_{0}$.

The transmission of arterial pulsations through the CSF is also linked to the windkessel effect [45]. This mechanism involves a progressive dampening of arterial pulsations, mostly through the CSF, in order to reach the capillary bed of the brain as a nonpulsatile flow $[45,46]$. In normal cerebral blood flow this occurs as a consequence of a close coupling between CSF oscillations and arterial pulsations, which leads to a resonance state [46]. However, alterations in NPH cause that CSF pulsations shift out from resonance $[45,46]$. 
Consequently, the arterial pulse pressure transmitted to the capillary circulation would be stronger [45]. In this study, we observed that the state of intracranial hypertension induced by ITs affects the ABP component of the ICP waveform (band $B_{2}$ ), which is consistent with a disruption of the windkessel effect triggered by infusion.

Finally, some authors have suggested that elevated ICP may activate an intracranial baroreflex conducted through the autonomic system [47]. The moderate rise in ICP during ITs may result in a reversible pressure-driven systemic response. This produces an elevation in $\mathrm{ABP}$ and heart rate variance, as well as a decrease in cerebral perfusion pressure (CPP) and blood flow velocity (FV) [47]. The changes in ABP as a consequence of a moderate rise in ICP are compatible with an early Cushing response [47]. The differences in band $B_{2}$ between $E_{2}$ and $E_{0}$ may reflect the influence of the moderate hypertension produced during ITs in systemic haemodynamics. Our results could be indicative of an adaptive haemodynamic response modulated by the presence of an intracranial baroreflex [47].

The statistically significant differences between phases of the IT were mainly found in $B_{2}$, which leads us to hypothesise that the previous changes affect mainly the pulse component of the ICP waveform. However, some statistically significant differences were also observed in band $B_{1}$, related to the respiratory-related component of the ICP waveform. Previous investigations also reported a relationship between pressure changes and the respiratory component of the ICP waveform $[25,48]$. It has been shown that, under reduced pressurevolume compliance conditions, ventilatory alternations in cerebral FV are reduced $[25,48]$, while ICP appears to be unaffected [25]. Our results using $\operatorname{SD}\left[W T_{B_{1}}\right]$ suggest that there is a significantly higher variability in the spectral content of band $B_{l}$ when CSF pressure reaches the range of intracranial hypertension. However, results were not as significant as in $B_{2}$. The methodologies used in previous studies were very different from ours, and included 
experimental models [48] and evoked respiratory waves [25]. This may be the reason why our results show a weaker link between ICP and respiratory waves.

\subsection{Relationship between wavelet parameters and patient data}

We also analysed the correlation between wavelet parameters and several demographic, radiological and ICP-based variables extracted for the patients in our database. Results in Table 4 show that a few significant correlations could be found. The most significant correlations were related to the amplitude of the signal in the plateau phase $\left(A_{p}\right)$. It is noteworthy that, in the case of $\left\langle W T_{B_{2}}\right\rangle$, significant correlations with $A_{p}$ were found in all the artefact-free epochs of the infusion study. Besides, for $\operatorname{SD}\left[W T_{B_{2}}\right]$, significant correlations with $A_{p}$ were found for the basal, early infusion and recovery phases. These results suggest that the changes in the average degree of similarity in the spectral content and the variability of the ICP signal in band $B_{2}$ are linked to changes in signal amplitude produced by intracranial hypertension induced by ITs.

\subsection{Limitations of the study and future research lines}

Finally, some issues merit consideration. Firstly, it should be noted that the ICP waveform has a third component: slow waves $(0.0055-0.05 \mathrm{~Hz})$ [11]. The frequency resolution associated with the complex Morlet wavelet has not allowed us to analyse this frequency range. Very low frequency ranges have been previously analysed using the wavelet transform, for example in the context of near-infrared spectroscopy signals [49] or blood flow velocity and arterial blood pressure investigation [32]. The analysis of very low frequencies is possible in these studies because the recording time is generally longer. Nonetheless, to the best of our knowledge, the COI was not considered. Additionally, slow waves are usually analysed in longer ICP signals and their study may be clinically relevant, since there is evidence that a 
frequent occurrence of these waves could be related to a positive response to shunting [19]. In ITs, recordings are shorter [11]. However, further investigations should be performed to certify whether very low frequency components could provide additional information about the mechanisms of cerebral autoregulation. Similarly, it could be interesting to study the frequency range above $5 \mathrm{~Hz}$ in order to analyse whether the harmonics of the fundamental frequency associated to the pulse wave may influence the calculation of parameters derived from the wavelet transform. It should also be considered that, in this study, brain compliance has been estimated as a baseline RAP index. Further compliance estimators should be analysed in order to assess whether they could me more suitable for our recordings. Another important issue concerns the cohort of subjects enrolled in the study. Although all the patients showed clinical and radiological features of NPH, the mechanisms leading to hydrocephalus were diverse. Since this study is focused on the wavelet characterisation of ICP signals, we consider that patient heterogeneity should not be regarded as an important drawback. Additionally, it would be desirable to analyse a larger dataset of ICP signals from ITs. In this sense, it would be interesting to compare $W E$ and $W T$ in healthy and hydrocephalic subjects. However, we believe that, for this task, it would be necessary to create a database of ICP recordings obtained less invasively than with ITs. Finally, the potential clinical applications of wavelet-derived parameters in ICP signal analysis need to be further explored. This is a first exploratory study, but the possible use of these measures may include the prediction of outcome after traumatic brain injury or the prediction of the response of hydrocephalus patients to shunting. We will also try to address real-time applications in future studies by using wavelet-related tools with lower computational cost than the CWT, like the discrete wavelet transform or wavelet packets. 


\subsection{Conclusion}

Wavelet parameters like $W T$ and $W E$ revealed changes in the signal time-scale representation during ITs. Our results show a higher degree of similarity in the spectral content of ICP signals, as well as a lower irregularity and variability in the plateau phase with respect to the basal phase in band $B_{2}$. We also found statistically significant differences between $E_{2}$ and $E_{0}$ for band $B_{1}$ using $\mathrm{SD}[W T]$.

\section{ACKNOWLEDGEMENTS}

This study was supported by the project TEC2014-53196-R from "Ministerio de Economía y Competitividad" and FEDER and the project VA059U13 from the "Consejería de Educación de la Junta de Castilla y León”. A. Bachiller was in receipt of a PIF-UVA grant from the University of Valladolid. 


\section{REFERENCES}

[1] M. Bergsneider, C. Miller, Surgical management of adult hydrocephalus, Surgery. 62 (2008) 643-660.

[2] R.A. Weerakkody, M. Czosnyka, M.U. Schuhmann, E.A. Schmidt, N. Keong, T. Santarius, et al., Clinical assessment of cerebrospinal fluid dynamics in hydrocephalus. Guide to interpretation based on observational study, Acta Neurol. Scand. 124 (2011) $85-98$.

[3] Y. Serulle, H. Rusinek, I.I. Kirov, H. Milch, E. Fieremans, A.B. Baxter, et al., Differentiating shunt-responsive normal pressure hydrocephalus from Alzheimer disease and normal aging: pilot study using automated MRI brain tissue segmentation., J. Neurol. 261 (2014) 1994-2002.

[4] A. Chari, M. Czosnyka, H.K. Richards, J.D. Pickard, Z.H. Czosnyka, Hydrocephalus shunt technology: 20 years of experience from the Cambridge Shunt Evaluation Laboratory., J. Neurosurg. 120 (2014) 697-707.

[5] N. Lenfeldt, A. Larsson, L. Nyberg, M. Andersson, R. Birgander, A. Eklund, et al., Idiopathic normal pressure hydrocephalus: increased supplementary motor activity accounts for improvement after CSF drainage., Brain. 131 (2008) 2904-12.

[6] P.K. Eide, Cardiac output in idiopathic normal pressure hydrocephalus: association with arterial blood pressure and intracranial pressure wave amplitudes and outcome of shunt surgery., Fluids Barriers CNS. 8 (2011) 11.

[7] M. Czosnyka, Z.H. Czosnyka, S. Momjian, J.D. Pickard, Cerebrospinal fluid dynamics., Physiol. Meas. 25 (2004) R51-R76.

[8] D. Santamarta, R. Hornero, D. Abásolo, M. Martínez-Madrigal, J. Fernández, J. García-Cosamalón, Complexity analysis of the cerebrospinal fluid pulse waveform during infusion studies, Child's Nerv. Syst. 26 (2010) 1683-1689.

[9] A. Eklund, P. Smielewski, I. Chambers, N. Alperin, J. Malm, M. Czosnyka, et al., Assessment of cerebrospinal fluid outflow resistance, Med. Biol. Eng. Comput. 45 (2007) 719-735.

[10] A. Agren-Wilsson, A. Eklund, L.-O.D. Koskinen, A.T. Bergenheim, J. Malm, Brain energy metabolism and intracranial pressure in idiopathic adult hydrocephalus syndrome., J. Neurol. Neurosurg. Psychiatry. 76 (2005) 1088-1093.

[11] S. Momjian, Z.H. Czosnyka, M. Czosnyka, J.D. Pickard, Link between vasogenic waves of intracranial pressure and cerebrospinal fluid outflow resistance in normal pressure hydrocephalus., Br. J. Neurosurg. 18 (2004) 56-61.

[12] M. Czosnyka, J.D. Pickard, Monitoring and interpretation of intracranial pressure., J. Neurol. Neurosurg. Psychiatry. 75 (2004) 813-821.

[13] R. Hornero, M. Aboy, D. Abásolo, J. McNames, B. Goldstein, Interpretation of approximate entropy: Analysis of intracranial pressure approximate entropy during acute intracranial hypertension, IEEE Trans. Biomed. Eng. 52 (2005) 1671-1680.

[14] C.-W. Lu, M. Czosnyka, J.-S. Shieh, A. Smielewska, J.D. Pickard, P. Smielewski, Complexity of intracranial pressure correlates with outcome after traumatic brain 
injury., Brain. 135 (2012) 2399-408.

[15] R. Hornero, M. Aboy, D. Abásolo, Analysis of intracranial pressure during acute intracranial hypertension using Lempel-Ziv complexity: Further evidence, Med. Biol. Eng. Comput. 45 (2007) 617-620.

[16] J.J. Lemaire, J.Y. Boire, J. Chazal, B. Irthum, A computer software for frequential analysis of slow intracranial pressure waves, Comput. Methods Programs Biomed. 42 (1994) 1-14.

[17] S. Holm, P.K. Eide, The frequency domain versus time domain methods for processing of intracranial pressure (ICP) signals, Med. Eng. Phys. 30 (2008) 164-170.

[18] M. García, J. Poza, D. Santamarta, D. Abásolo, P. Barrio, R. Hornero, Spectral analysis of intracranial pressure signals recorded during infusion studies in patients with hydrocephalus., Med. Eng. Phys. 35 (2013) 1490-8.

[19] M. Kasprowicz, S. Asgari, M. Bergsneider, M. Czosnyka, R. Hamilton, X. Hu, Pattern recognition of overnight intracranial pressure slow waves using morphological features of intracranial pressure pulse, J. Neurosci. Methods. 190 (2010) 310-318.

[20] X. Hu, P. Xu, F. Scalzo, P. Vespa, M. Bergsneider, Morphological Clustering and Analysis of Continuous Intracranial Pressure, IEEE Trans. Biomed. Eng. 56 (2009) 696-705.

[21] I.M. Elixmann, M. Kwiecien, C. Goffin, M. Walter, B. Misgeld, M. Kiefer, et al., Control of an electromechanical hydrocephalus shunt--a new approach., IEEE Trans. Biomed. Eng. 61 (2014) 2379-88.

[22] M. Latka, W. Kolodziej, M. Turalska, D. Latka, W. Zub, B.J. West, Wavelet assessment of cerebrospinal compensatory reserve and cerebrovascular reactivity., Physiol. Meas. 28 (2007) 465-479.

[23] H.E. Heissler, K. König, J.K. Krauss, E. Rickels, Stationarity in Neuromonitoring Data, Acta Neurochir. Suppl. 114 (2012) 93-95.

[24] P. Xu, X. Hu, D. Yao, Improved wavelet entropy calculation with window functions and its preliminary application to study intracranial pressure, Comput. Biol. Med. 43 (2013) 425-433.

[25] C. Haubrich, R.R. Diehl, M. Kasprowicz, J. Diedler, E. Sorrentino, P. Smielewski, et al., Traumatic brain injury: Increasing ICP attenuates respiratory modulations of cerebral blood flow velocity, Med. Eng. Phys. 37 (2015) 175-179.

[26] O. Rioul, M. Vetterli, Wavelets and signal processing, IEEE Signal Process. Mag. 8 (1991) 14-38.

[27] C. Torrence, G.P. Compo, A Practical Guide to Wavelet Analysis, Bull. Am. Meteorol. Soc. 79 (1998) 61-78.

[28] S. Mallat, A Wavelet Tour of Signal Processing: The Sparse Way, Academic Press, 2008.

[29] B.J. Roach, D.H. Mathalon, Event-related EEG time-frequency analysis: An overview of measures and an analysis of early gamma band phase locking in schizophrenia, Schizophr. Bull. 34 (2008) 907-926.

[30] A. Bachiller, J. Poza, C. Gómez, V. Molina, V. Suazo, R. Hornero, A comparative 
study of event-related coupling patterns during an auditory oddball task in schizophrenia., J. Neural Eng. 12 (2015) 016007.

[31] J. Poza, C. Gómez, M. García, R. Corralejo, A. Fernández, R. Hornero, Analysis of neural dynamics in mild cognitive impairment and Alzheimer's disease using wavelet turbulence., J. Neural Eng. 11 (2014) 026010.

[32] M. Turalska, M. Latka, K. Pierzchala, B.J. West, M. Czosnyka, Generation of very low frequency cerebral blood flow fluctuations in humans, Acta Neurochir. Suppl. 102 (2008) 43-47.

[33] P.R.B. Benchimol-Barbosa, A. de Souza-Bomfim, E. Corrêa-Barbosa, P. Ginefra, S.H. Cardoso-Boghossian, C. Destro, et al., Spectral turbulence analysis of the signalaveraged electrocardiogram of the atrial activation as predictor of recurrence of idiopathic and persistent atrial fibrillation, Int. J. Cardiol. 107 (2006) 307-316.

[34] J.W. Sleigh, D.A. Steyn-Ross, M.L. Steyn-Ross, C. Grant, G. Ludbrook, Cortical entropy changes with general anaesthesia: theory and experiment, Physiol. Meas. 25 (2004) 921-934.

[35] M. García, J. Poza, D. Abásolo, D. Santamarta, R. Hornero, Analysis of intracranial pressure signals recorded during infusion studies using the spectral entropy., in: Proc. Annu. Int. Conf. IEEE Eng. Med. Biol. Soc., 2013: pp. 2543-6.

[36] J.D. Jobson, Applied Multivariate Data Analysis, Springer New York, New York, NY, 1991.

[37] D.J. Kim, Z. Czosnyka, N. Keong, D.K. Radolovich, P. Smielewski, M.P.F. Sutcliffe, et al., Index of cerebrospinal compensatory reserve in hydrocephalus, Neurosurgery. 64 (2009) 494-502.

[38] M. Aboy, J. McNames, W. Wakeland, B. Goldstein, Pulse and mean intracranial pressure analysis in pediatric traumatic brain injury, in: Intracranial Press. Brain Monit. XII, Springer-Verlag, Vienna, 2005: pp. 307-310.

[39] J. Poza, R. Hornero, D. Abásolo, A. Fernández, M. García, Extraction of spectral based measures from MEG background oscillations in Alzheimer's disease, Med. Eng. Phys. 29 (2007) 1073-1083.

[40] D. Santamarta, D. Abásolo, M. Martínez-Madrigal, R. Hornero, Characterisation of the intracranial pressure waveform during infusion studies by means of central tendency measure, Acta Neurochir. (Wien). 154 (2012) 1595-1602.

[41] G.A. Bateman, Vascular compliance in normal pressure hydrocephalus, Am. J. Neuroradiol. 21 (2000) 1574-1585.

[42] M.E. Wagshul, E.J. Kelly, H.J. Yu, B. Garlick, T. Zimmerman, M.R. Egnor, Resonant and notch behavior in intracranial pressure dynamics, J. Neurosurg. Pediatr. 3 (2009) 354-364.

[43] C. Haubrich, Z.H. Czosnyka, A. Lavinio, P. Smielewski, R.R. Diehl, J.D. Pickard, et al., Is there a direct link between cerebrovascular activity and cerebrospinal fluid pressure-volume compensation?, Stroke. 38 (2007) 2677-2680.

[44] B.J.E. Misgeld, R. Mondal, S. Leonhardt, Pulsatile cerebrospinal model with cardiovascular coupling, IFAC-PapersOnLine. 48 (2015) 183-188.

[45] M. Egnor, L. Zheng, A. Rosiello, F. Gutman, R. Davis, A model of pulsations in 
communicating hydrocephalus, Pediatr. Neurosurg. 36 (2002) 281-303.

[46] G.A. Bateman, C.R. Levi, P. Schofield, Y. Wang, E.C. Lovett, The venous manifestations of pulse wave encephalopathy: windkessel dysfunction in normal aging and senile dementia., Neuroradiology. 50 (2008) 491-7.

[47] E.A. Schmidt, Z.H. Czosnyka, S. Momjian, M. Czosnyka, R.A. Bech, J.D. Pickard, Intracranial baroreflex yielding an early Cushing response in human, Acta Neurochir. Suppl. 95 (2005) 253-256.

[48] X. Hu, A.A. Alwan, E.H. Rubinstein, M. Bergsneider, Reduction of compartment compliance increases venous flow pulsatility and lowers apparent vascular compliance: implications for cerebral blood flow hemodynamics., Med. Eng. Phys. 28 (2006) 30414.

[49] P.S. Addison, A Review of Wavelet Transform Time-Frequency Methods for NIRSBased Analysis of Cerebral Autoregulation., IEEE Rev. Biomed. Eng. 8 (2015) 78-85. 


\section{TABLES}

Table 1. Data recorded from the subjects under study.

Table 2. Median [interquartile range, IQR] values of the epoch length and CSF pressure.

Table 3. $Z$ statistics and $p$-values associated with the Wilcoxon signed-rank tests. The significant values ( $p<1.70 \cdot 10^{-3}$, Bonferroni-corrected $)$ are highlighted.

Table 4. Correlation coefficients, $r$, and $p$-values obtained for the Spearman test to determine the correlation between wavelet parameters and patient data. Significant correlations $(p<0.01)$ are highlighted. 


\section{FIGURE LEGENDS}

Figure 1. Evolution of the CSF pressure during the infusion test for a patient diagnosed with normal pressure hydrocephalus. The four artefact-free epochs selected by a neurosurgeon have been indicated ( $E_{0}$ : epoch $0, E_{1}$ : epoch $1, E_{2}$ : epoch $2, E_{3}$ : epoch 3$)$.

Figure 2. Scalogram obtained for the ICP recording of Fig. 1. The transparency outline delineates the limits of the cone of influence (COI), where border effects can be ignored. The black horizontal lines indicate the limits of frequency bands $B_{1}(0.15-0.3 \mathrm{~Hz})$ and $B_{2}(0.67-$ $2.5 \mathrm{~Hz})$.

Figure 3. Boxplots displaying the distribution of $\langle W T\rangle$ and $\mathrm{SD}[W T]$ for frequency bands $B_{1}$ and $B_{2}$ in the four artefact-free epochs. (a) $<W T_{B_{1}}>$. (b) $\operatorname{SD}\left[W T_{B_{1}}\right]$. (c) $<W T_{B_{2}}>$. (d) $\mathrm{SD}\left[W T_{B_{2}}\right]$. The statistically significant differences are indicated with an asterisk (* $p<1.70 \cdot 10^{-3}$, Bonferroni-corrected).

Figure 4. Boxplots displaying the distribution of $\langle W E\rangle$ and $\operatorname{SD}[W E]$ for frequency bands $B_{1}$ and $B_{2}$ in the four artefact-free epochs. (a) $<W E_{B_{1}}>$. (b) $\mathrm{SD}\left[W E_{B_{1}}\right]$. (c) $<W E_{B_{2}}>$. (d) $\mathrm{SD}\left[W E_{B_{2}}\right]$. The statistically significant differences are indicated with an asterisk (* $p<1.70 \cdot 10^{-3}$, Bonferroni-corrected). 
Table 1. Data recorded from the subjects under study.

\begin{tabular}{cc}
\hline Characteristic & $\begin{array}{c}\text { Value } \\
\text { (median }[\mathrm{IQR}])\end{array}$ \\
\hline Number of subjects $(n)$ & 112 \\
Age (years) & $74[63-80]$ \\
Ventricular size (Evans index, $E)$ & $0.37[0.35-0.41]$ \\
Basal pressure $\left(P_{0}\right)(\mathrm{mm} \mathrm{Hg})$ & $7.71[5.53-11.10]$ \\
Basal amplitude $\left(A_{0}\right)(\mathrm{mm} \mathrm{Hg})$ & $2.73[1.57-3.46]$ \\
Plateau pressure $\left(P_{p}\right)(\mathrm{mm} \mathrm{Hg})$ & $24.95[18.57-32.67]$ \\
Plateau amplitude $\left(A_{p}\right)(\mathrm{mm} \mathrm{Hg})$ & $9.74[5.93-13.85]$ \\
Outflow resistance $(R)\left(\mathrm{mm} \mathrm{Hg} \mathrm{ml}{ }^{-1} \mathrm{~min}\right)$ & $11.21[7.34-14.98]$
\end{tabular}

IQR: interquartile range 
Table 2. Median [interquartile range, IQR] values of the epoch length and CSF pressure.

\begin{tabular}{ccccc}
\hline & Epoch 0 & Epoch 1 & Epoch 2 & Epoch 3 \\
\hline Length (s) & $156[120-180]$ & $300[240-330]$ & $480[360-600]$ & $180[130-205]$ \\
$\begin{array}{c}\text { CSF pressure } \\
(\mathrm{mm} \mathrm{Hg})\end{array}$ & $7.71[5.53-11.10]$ & $16.10[12.28-20.75]$ & $24.95[18.57-32.67]$ & $15.36[11.62-19.50]$ \\
\hline CSF: cerebrospinal fluid & & &
\end{tabular}


Table 3. $Z$ statistics and $p$-values associated with the Wilcoxon signed-rank tests. The significant values $\left(p<1.70 \cdot 10^{-3}\right.$, Bonferroni-corrected) are highlighted.

\begin{tabular}{|c|c|c|c|c|c|c|c|c|c|c|c|c|}
\hline & \multicolumn{2}{|c|}{$E_{0}$ vs. $E_{l}$} & \multicolumn{2}{|c|}{$E_{0}$ vs. $E_{2}$} & \multicolumn{2}{|c|}{$E_{0}$ vs. $E_{3}$} & \multicolumn{2}{|c|}{$E_{1}$ vs. $E_{2}$} & \multicolumn{2}{|c|}{$E_{1}$ vs. $E_{3}$} & \multicolumn{2}{|c|}{$E_{2}$ vs. $E_{3}$} \\
\hline & $Z$ & $p$ & $Z$ & $p$ & $Z$ & $p$ & $Z$ & $p$ & $Z$ & $p$ & $Z$ & $p$ \\
\hline$<W T_{B_{1}}>$ & -2.85 & $4.40 \cdot 10^{-3}$ & -2.97 & $3.01 \cdot 10^{-3}$ & -1.66 & $9.68 \cdot 10^{-2}$ & -2.42 & $1.55 \cdot 10^{-2}$ & -0.50 & 0.62 & -2.73 & $6.24 \cdot 10^{-3}$ \\
\hline$<W T_{B_{2}}>$ & -6.13 & $8.71 \cdot 10^{-10}$ & -7.80 & $6.16 \cdot 10^{-15}$ & -1.42 & $1.14 \cdot 10^{-13}$ & -3.06 & $2.21 \cdot 10^{-3}$ & -0.38 & 0.71 & -2.85 & $4.44 \cdot 10^{-3}$ \\
\hline $\mathrm{SD}\left[W T_{B_{2}}\right]$ & -5.82 & $5.86 \cdot 10^{-9}$ & -6.90 & $5.17 \cdot 10^{-12}$ & -7.04 & $1.96 \cdot 10^{-12}$ & -1.63 & 0.10 & -1.17 & 0.24 & -0.61 & 0.54 \\
\hline$<W E_{B_{2}}>$ & -6.05 & $1.45 \cdot 10^{-9}$ & -6.15 & $7.95 \cdot 10^{-10}$ & -5.40 & $6.56 \cdot 10^{-8}$ & -1.80 & $7.19 \cdot 10^{-2}$ & -1.08 & 0.28 & -2.94 & $3.27 \cdot 10^{-3}$ \\
\hline $\mathrm{SD}\left[W E_{B_{2}}\right]$ & -6.77 & $1.29 \cdot 10^{-11}$ & -7.30 & $2.91 \cdot 10^{-13}$ & -7.49 & $6.88 \cdot 10^{-14}$ & -5.48 & $4.30 \cdot 10^{-8}$ & -4.02 & $5.87 \cdot 10^{-5}$ & -1.70 & $8.95 \cdot 10^{-2}$ \\
\hline
\end{tabular}

$E_{0}$ : epoch $0 ; E_{1}$ : epoch $1 ; E_{2}$ : epoch $2 ; E_{3}$ : epoch $3 ;<W T_{B_{1}}>:$ mean wavelet turbulence in band $B_{1} ; \operatorname{SD}\left[W T_{B_{1}}\right]:$ standard deviation of the wavelet turbulence in band $B_{1} ;\left\langle W T_{B_{2}}\right\rangle$ : mean wavelet turbulence in band $B_{2} ; \operatorname{SD}\left[W T_{B_{2}}\right]:$ standard deviation of the wavelet turbulence in band $B_{2} ;<W E_{B_{1}}>$ : mean wavelet entropy in band $B_{1} ;<W E_{B_{2}}>$ : mean wavelet entropy in band $B_{2}$; $\mathrm{SD}\left[W E_{B_{2}}\right]:$ standard deviation of the wavelet entropy in band $B_{2}$. 
Table 4.-. Correlation coefficients, $r$, and $p$-values obtained for the Spearman test to determine correlation between wavelet parameters and patient data. Values with a significance level $p<0.01$ are highlighted.

\begin{tabular}{|c|c|c|c|c|c|c|c|c|}
\hline & \multicolumn{2}{|c|}{$A_{0}$} & \multicolumn{2}{|c|}{$A_{p}$} & \multicolumn{2}{|c|}{$R_{\text {OUT }}$} & \multicolumn{2}{|c|}{$R A P$} \\
\hline & $r$ & $p$ & $r$ & $p$ & $r$ & $p$ & $r$ & $p$ \\
\hline$<W T_{B_{1}}^{E_{0}}>$ & 0.1789 & 0.0592 & -0.1717 & 0.0703 & -0.1310 & 0.1685 & $-0,0256$ & 0,7889 \\
\hline$<W T_{B_{1}}^{E_{1}}>$ & 0.0148 & 0.8767 & -0.1780 & 0.0604 & -0.1285 & 0.1768 & $-0,0448$ & 0,6393 \\
\hline$<W T_{B_{1}}^{E_{2}}>$ & -0.1020 & 0.2844 & -0.3158 & 0.0007 & -0.2285 & 0.0154 & 0,0502 & 0,5995 \\
\hline$<W T_{B_{1}}^{E_{3}}>$ & 0.0386 & 0.6863 & -0.0918 & 0.3355 & -0.0528 & 0.5803 & 0,0149 & 0,8763 \\
\hline $\mathrm{SD}\left[W T_{B_{1}}^{E_{0}}\right]$ & -0.1712 & 0.0712 & 0.1833 & 0.0530 & 0.1450 & 0.1271 & 0,0517 & 0,5886 \\
\hline $\mathrm{SD}\left[W T_{B_{1}}^{E_{1}}\right]$ & -0.0236 & 0.8049 & 0.1143 & 0.2302 & 0.0772 & 0.4184 & 0,0742 & 0,4371 \\
\hline $\mathrm{SD}\left[W T_{B_{1}}^{E_{2}}\right]$ & 0.1044 & 0.2732 & 0.2728 & 0.0036 & 0.1883 & 0.0468 & $-0,0327$ & 0,7321 \\
\hline $\mathrm{SD}\left[W T_{B_{1}}^{E_{3}}\right]$ & -0.0003 & 0.9973 & 0.0772 & 0.4185 & 0.0471 & 0.6218 & $-0,0262$ & 0,7841 \\
\hline$<W T_{B_{2}}^{E_{0}}>$ & 0.3389 & 0.0003 & 0.4347 & $1.68 \cdot 10^{-6}$ & 0.1997 & 0.0347 & $-0,1535$ & 0,1061 \\
\hline$<W T_{B_{2}}^{E_{1}}>$ & 0.1804 & 0.0570 & 0.4050 & $9.43 \cdot 10^{-6}$ & 0.1984 & 0.0360 & $-0,0813$ & 0,3943 \\
\hline$<W T_{B_{2}}^{E_{2}}>$ & 0.1165 & 0.2210 & 0.2762 & 0.0032 & 0.0808 & 0.3971 & 0,0262 & 0,7835 \\
\hline$<W T_{B_{2}}^{E_{3}}>$ & 0.1012 & 0.2885 & 0.3818 & $3.28 \cdot 10^{-5}$ & 0.1843 & 0.0518 & $-0,0190$ & 0,8422 \\
\hline $\mathrm{SD}\left[W T_{B_{2}}^{E_{0}}\right]$ & -0.3081 & 0.0010 & -0.3626 & $8.55 \cdot 10^{-5}$ & -0.1384 & 0.1455 & 0,2129 & 0,0242 \\
\hline $\mathrm{SD}\left[W T_{B_{2}}^{E_{1}}\right]$ & -0.1979 & 0.0365 & -0.3762 & $4.35 \cdot 10^{-5}$ & -0.1968 & 0.0375 & 0,1271 & 0,1818 \\
\hline $\mathrm{SD}\left[W T_{B_{2}}^{E_{2}}\right]$ & -0.0905 & 0.3426 & -0.1970 & 0.0374 & -0.0573 & 0.5483 & $-0,0190$ & 0,8421 \\
\hline $\mathrm{SD}\left[W T_{B_{2}}^{E_{3}}\right]$ & -0.1099 & 0.2486 & -0.2919 & 0.0018 & -0.1378 & 0.1475 & 0,0828 & 0,3854 \\
\hline$<W E_{B_{1}}^{E_{0}}>$ & -0.0381 & 0.6901 & 0.0490 & 0.6081 & 0.0413 & 0.6657 & 0,1055 & 0,2684 \\
\hline$<W E_{B_{1}}^{E_{1}}>$ & -0.0832 & 0.3829 & 0.0084 & 0.9298 & -0.0873 & 0.3602 & 0,1881 & 0,0470 \\
\hline$<W E_{B_{1}}^{E_{2}}>$ & 0.0045 & 0.9625 & 0.0213 & 0.8234 & -0.0621 & 0.5152 & 0,0910 & 0,3402 \\
\hline$<W E_{B_{1}}^{E_{3}}>$ & -0.0459 & 0.6306 & -0.0026 & 0.9783 & -0.0688 & 0.4710 & 0,1248 & 0,1900 \\
\hline $\mathrm{SD}\left[W E_{B_{1}}^{E_{0}}\right]$ & 0.0821 & 0.3892 & 0.1187 & 0.2126 & 0.0684 & 0.4734 & $-0,0329$ & 0,7303 \\
\hline $\mathrm{SD}\left[W E_{B_{1}}^{E_{1}}\right]$ & 0.1398 & 0.1417 & 0.0112 & 0.9068 & 0.0676 & 0.4788 & $-0,0574$ & 0,5477 \\
\hline $\mathrm{SD}\left[W E_{B_{1}}^{E_{2}}\right]$ & 0.0084 & 0.9301 & -0.0107 & 0.9105 & 0.0657 & 0.4914 & $-0,0747$ & 0,4338 \\
\hline $\mathrm{SD}\left[W E_{B_{1}}^{E_{3}}\right]$ & 0.0972 & 0.3078 & 0.0610 & 0.5231 & 0.0792 & 0.4062 & $-0,0924$ & 0,3327 \\
\hline$<W E_{B_{2}}^{E_{0}}>$ & -0.2471 & 0.0086 & -0.2933 & 0.0017 & -0.0730 & 0.4442 & 0,0094 & 0,9220 \\
\hline$<W E_{B_{2}}^{E_{1}}>$ & -0.0174 & 0.8554 & -0.2696 & 0.0040 & -0.0985 & 0.3013 & $-0,0752$ & 0,4304 \\
\hline$<W E_{B_{2}}^{E_{2}}>$ & 0.0189 & 0.8430 & -0.1994 & 0.0350 & -0.0575 & 0.5467 & $-0,0879$ & 0,3566 \\
\hline$<W E_{B_{2}}^{E_{3}}>$ & -0.0505 & 0.5973 & -0.3153 & 0.0007 & -0.1189 & 0.2119 & $-0,1011$ & 0,2887 \\
\hline $\mathrm{SD}\left[W E_{B_{2}}^{E_{0}}\right]$ & -0.2669 & 0.0044 & -0.3282 & 0.0004 & -0.2776 & 0.0030 & 0,1756 & 0,0640 \\
\hline $\mathrm{SD}\left[W E_{B_{2}}^{E_{1}}\right]$ & -0.1898 & 0.0451 & -0.1933 & 0.0411 & -0.2269 & 0.0161 & 0,2175 & 0,0212 \\
\hline $\mathrm{SD}\left[W E_{B_{2}}^{E_{2}}\right]$ & -0.1088 & 0.2535 & -0.0482 & 0.6139 & -0.1428 & 0.1332 & 0,1803 & 0,0571 \\
\hline $\mathrm{SD}\left[W E_{B_{2}}^{E_{3}}\right]$ & -0.1503 & 0.1138 & -0.1481 & 0.1193 & -0.2138 & 0.0236 & 0,1851 & 0,0507 \\
\hline
\end{tabular}




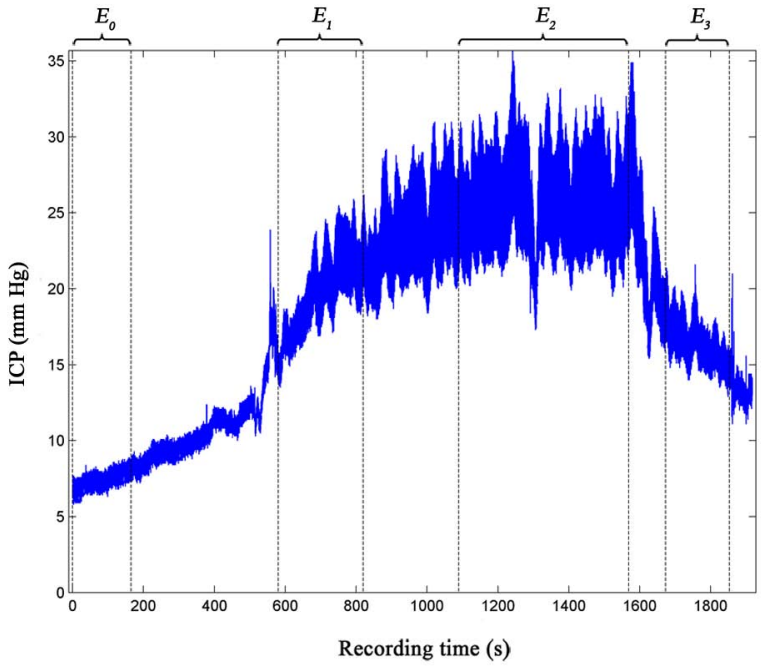




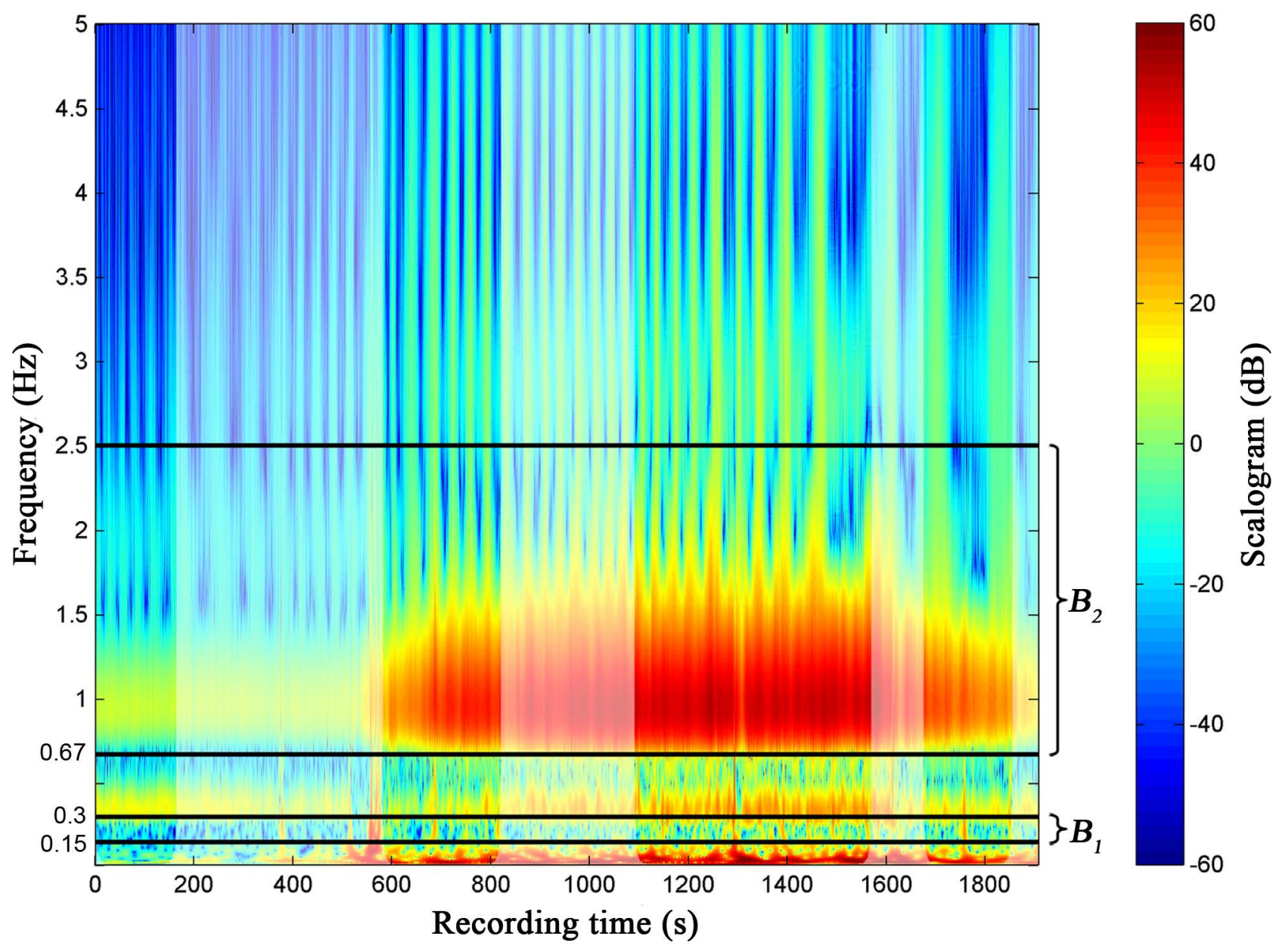


(a)

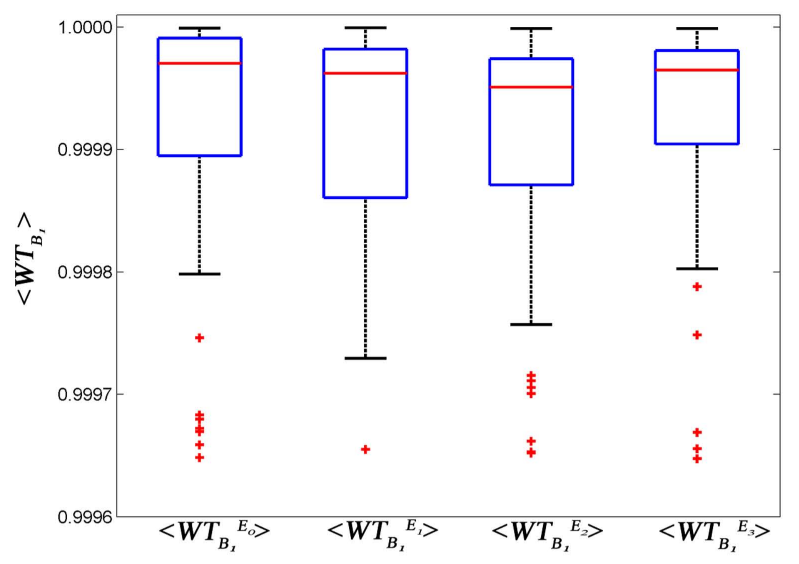

(c)

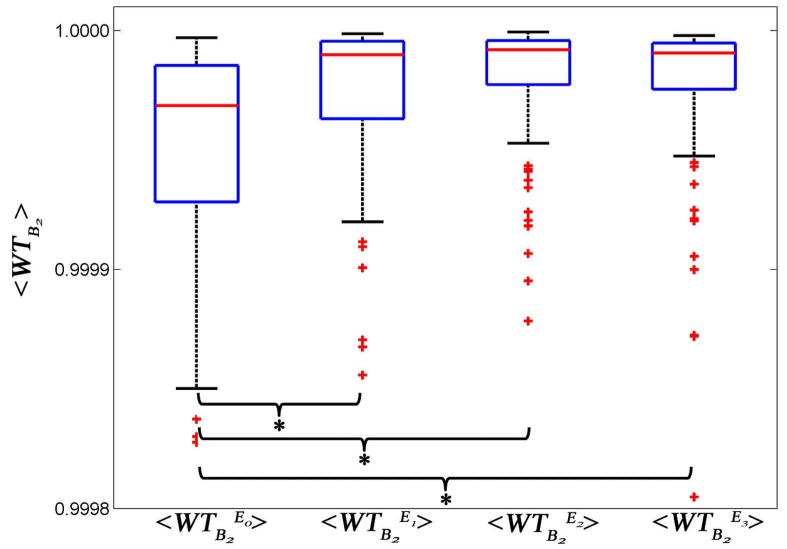

(b)

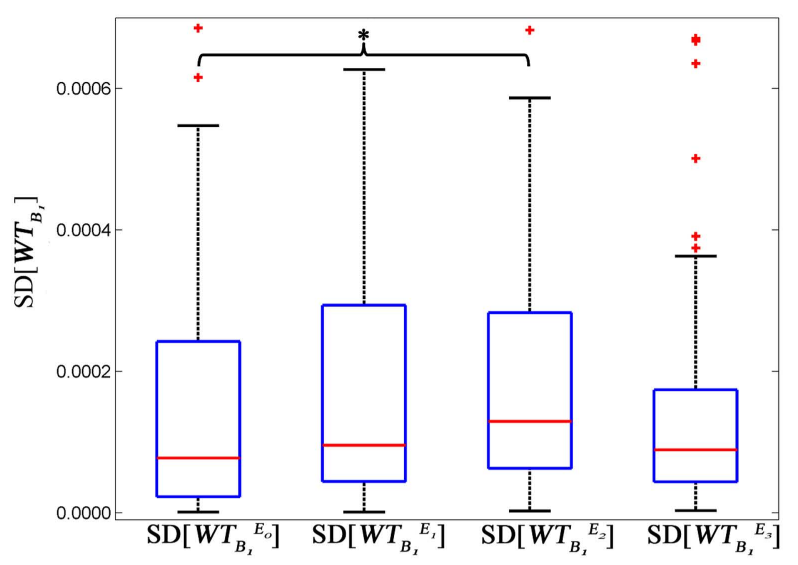

(d)

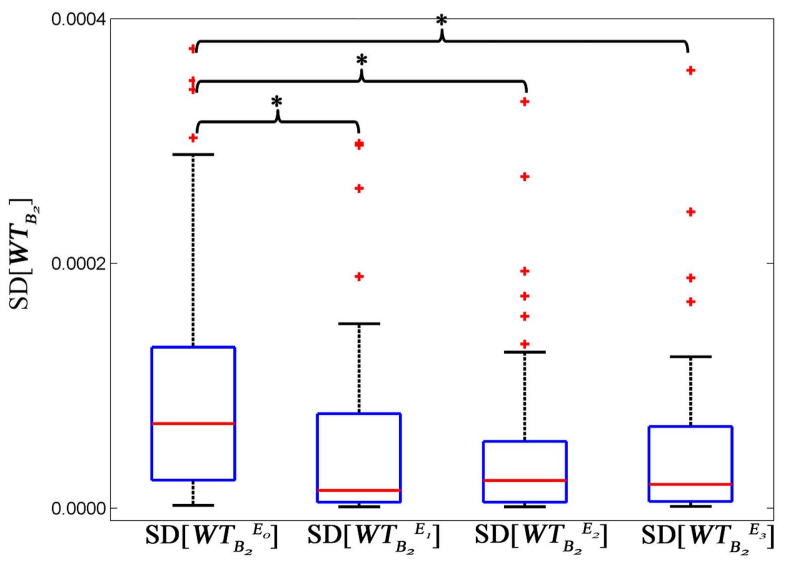


(a)

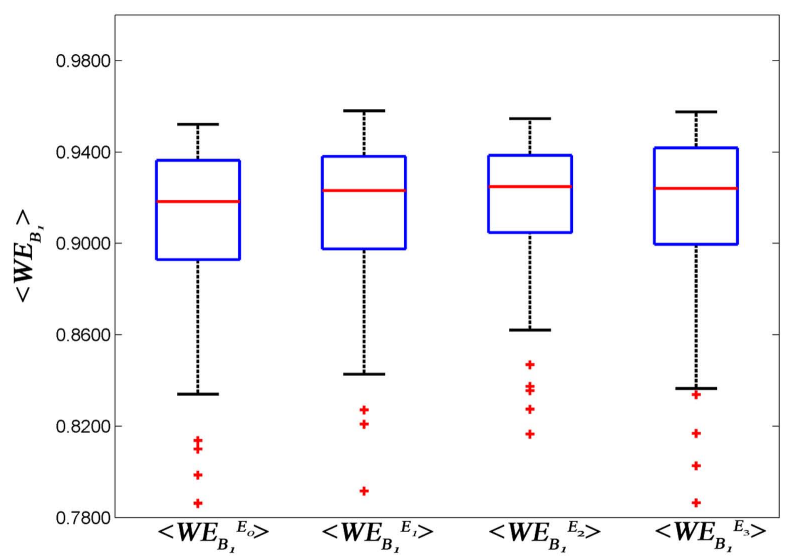

(c)

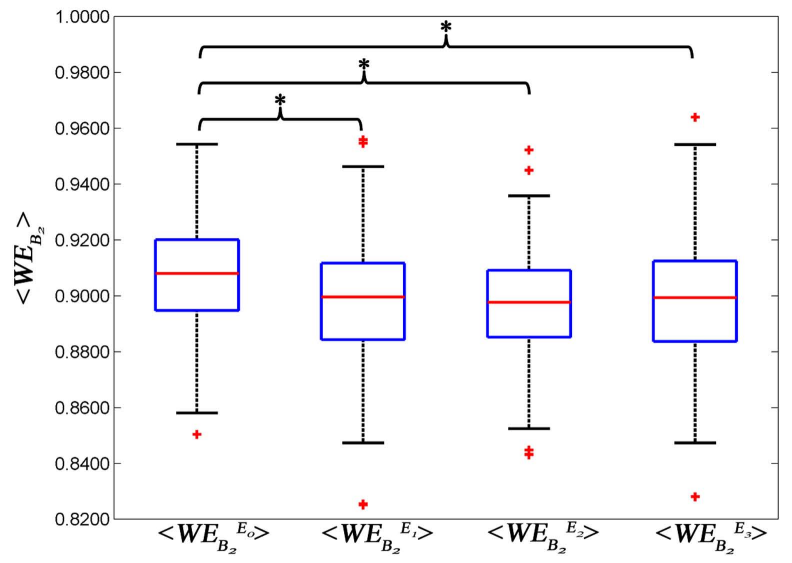

(b)

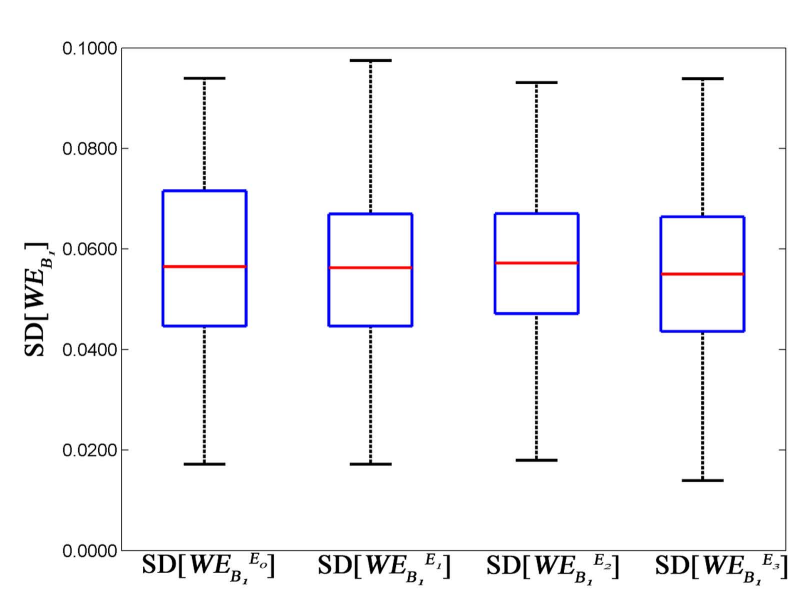

(d)

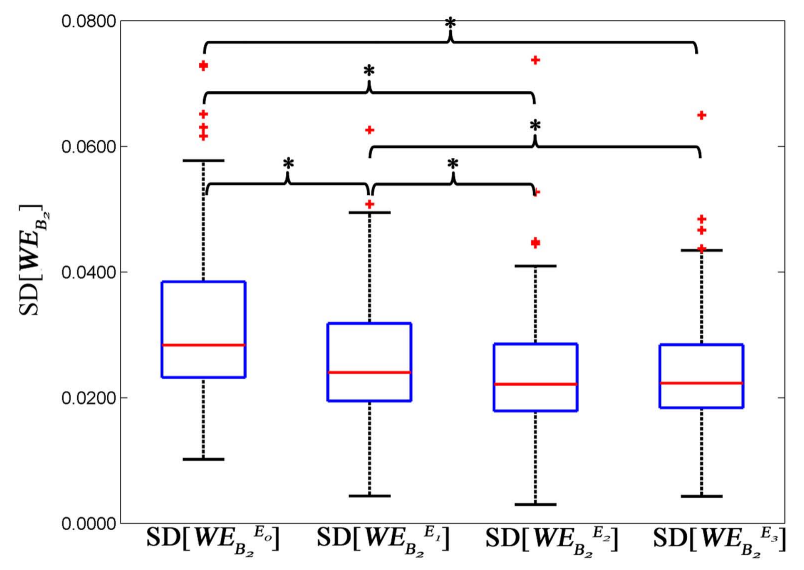

